\title{
When Choice Matters: Assortment and Participation for Performance on Digital Platforms
}

\author{
Prasanna P. Karhade \\ University of Hawai'i at Mānoa \\ karhade@hawaii.edu
}

\author{
Abhishek Kathuria \\ Indian School of Business \\ abhishek_kathuria@isb.edu
}

\author{
Benn R. Konsynski \\ Emory University \\ benn.konsynski@emory.edu
}

\begin{abstract}
Digital platforms are conveniently connecting multiple service providers with their consumers. How does assortment and participation influence the performance of service providers on digital platforms? In particular, food delivery platforms are providing consumers with a high level of home delivery convenience. Food delivery platforms are also unique as they provide a hybrid set of services to their consumers (both home delivery convenience and enable restaurant dining experience). The population of 95,735 restaurants on a pan India food discovery and delivery platform, serving a total of 135 different cuisines, located in 37 cities of India serves as our dataset. Ours is the first study to integrate induction and abduction to examine performance of service providers (restaurants) operating on a digital platform in a hybrid mode (providing both home delivery convenience and enabling restaurant dining experience). We find assortment matters and derive strong business implications for service science.
\end{abstract}

\section{Introduction}

The rapid proliferation of digital platforms over the past few years has transformed management practice and the nature of commerce and innovation [1-6]. Such platforms, which exemplify new age service systems, are co-evolving with customer expectations to evoke a paradigm wherein instead of bringing the customer to the enterprise, business models are evolving to bring the enterprise to the customer [7]. However, the notion of convenience as the new organizing principle is predicated upon participation of service providers on digital platforms. It is critical to note that platform participation is a strategic decision for service providers, offering complex opportunities and challenges [8]. Service providers must balance market access and market risk - while platform participation can provide access to new customers, platform-driven demand is fundamentally unpredictable with high holding or stockout costs [8]. Further, service providers must also ensure alignment of the platform participation decision with the value proposition offered to customers as some customers seek experience, rather than convenience.

A second critical strategic decision for service providers is the variety, or assortment of services offered. Formally, assortment size refers to the number of product or service categories that a seller carries or offers [9]. There is a widespread assumption that more variety is better; ergo, the more product or service categories offered by a seller, the better performance of the seller [10]. Hence, providers often offer a large assortment of products and services. Due to the inherent advantages of the digital medium, online sellers particularly often present a variety of offerings. However, despite the competitive advantage bestowed by large assortments, managing multiple product categories is a complex and risky endeavor [11]. Accordingly, the extent of variety offered by a seller is a complex strategic decision.

These two critical strategic decisions are often considered independent of one another. However, these two fundamental and complex decisions co-exist and have severe implications for the performance of service providers. Restaurants, in particular, strategically choose to provide home delivery convenience and at the same time also make significant investments to enhance restaurant dining experience. Both these choices are strategic to restaurants and have a significant influence on their performance. Hence, it is incumbent upon us to examine their concurrent effects. This reasoning leads us to our research question:

How do the decisions of participation on digital platforms and assortment of offerings concurrently influence the performance of service providers?

Through prior research has examined both questions individually, a concurrent examination is lacking. Further, most of these individual examinations, barring one exception [8], have been conducted at a single level of theory due to data and/or methodological limitations. However, given that there are multiple 
covariates (or information attributes) across multiple levels of theory that may contemporaneously influence the performance of service providers, a multi-level theoretical investigation is necessitated. Further, the performance of service providers, as assessed by customers, is the result of customer decision journeys, which encompass patterns of decision sequences. Ergo, the customer decision journey, consisting the partial orderings of its constituent decision points and forks are as critical as the final decision outcome of supplier performance itself $[1,12]$.

Guided by these logics, we address this research gap by leveraging a multi-level, multi-theoretic and multi-method approach that encapsulates notions of emergence in decision making logics [12]. We analyze a unique population-sized dataset using a sequence of induction and abduction in a data-driven analytics methodological approach. This method of inductive data-driven analytics followed by abductive discovery has been demonstrated to yield novel theoretical insights from large datasets by elegantly integrating multiple theoretical perspectives across multiple theoretical levels $[8,13]$.

Specifically, we use decision tree induction to identify patterns in the data, which consequently serve as inputs to the abduction process, whereby we generalize the patterns to the most plausible explanations. Decision trees shine the light on the flow of the decision-making process and model the customers' decision journeys constituting their cumulative experiences as they consume services offered by service providers [14]. These service providers are either focused on convenience seeking customers and participate on a digital platform or are focused on experience seeking customers and do not participate on the platform. Further, these service providers either offer large or small assortment of services. Our decision trees were grown using the $\mathrm{C} 4.5$ decision tree classification algorithm $[15,16]$ and were aggressively pruned to discover the underlying tacit structure of the data. Our large, population-level dataset and research setup enable us to leverage this method and benefit from its advantages, which include a low rate of false positive predictions [80]. We then apply abductive discovery, a third type of logical conclusion, generate novel insights regarding the concurrent influence of platform participation and variety on performance of service providers. In abduction, data is interpreted, with the aid of intellectual effort [17], to discover combinations of features for which there is no appropriate explanation in the store of extant knowledge [18]. This sequence of induction and abduction is appropriate for our investigation as a service provider performance is an encapsulation of customer decision journeys and combination of decision sequences that cannot be theorized ex-ante.

The specific context for our study is a digital platform that offers food discovery and delivery. Such platforms are an exemplar of a service system, wherein service providers (restaurants) who participate can fulfill needs of convenience seeking customers, which contrasts with non-participating service providers (restaurants) who seek to fulfill the needs of experience seeking customers. Such platforms have witnessed large investments as they serve large potential markets but are non-exclusive with minimal ability to lock-in users (due to low multi-homing costs) [8]. The empirical context is the entire population of (registered) restaurants in India. India, a fast-growing major economy, is an exemplar of a GREAT (growing, rural, eastern, aspirational, and transitional) domain [19], and serves as a setting for an increasing number of research studies (e.g., [8, 19-22]). The platform we study is a comprehensive review and rating site on which all restaurants are listed and receive ratings, irrespective of whether they participate in food delivery services. Thus, a key strength of this research design is that it addresses potential concerns stemming from sample selection bias.

Restaurants must explicitly decide whether to participate on the digital platform, which forms the first strategic decision made by restaurants. Further, the number of cuisines offered by the restaurant is form of product or service category assortment and serves as the second key strategic decision taken by the restaurants. Finally, the performance of restaurants is reflected by the ratings, which encapsulate customer journeys. Ratings are a specific type of aggregated user generated content and impact strategic behavior and decisionmaking of consumers. While reviews and user interactions capture many nuances [23], ratings reflect all these together in a single indicator.

The rest of this manuscript is organized as follows. In the next section we present an overview of related research and identify key thematic areas. Subsequently, we describe the tree induction methodology, the research context and the data used in the empirical investigation. We then elucidate the key information attributes present in our theory. Next, we showcase our computational experiments and step taken to perform induction. In the next section, we present findings from the sequence of decision tree induction and abduction. We conclude by discussing the implications of our findings and offering rich managerial implications of our research. Overall, we contribute to our theoretical understanding of how platform participation and variety concurrently influence the performance of service providers. 


\section{Related Literature}

\subsection{Digital platforms and Service Participation}

Digital platforms "facilitate transactions among firms and/or individuals who may not have been able to transact otherwise" [52, p. 141]. Note that as per the worldview of service-dominant logic (S-D logic), a service is the application of resources for the benefit of another [24]; a service provider hence applies resources for the benefit of another. A service system is a dynamic configuration of resources that creates value with other service systems through shared information [25]. Hence, digital platforms and their constituent subsystems (such as organizations who provide and consume services through the platform), which create value through shared information, are exemplars of service systems.

Digital platforms have stimulated the emergence of new business models [26], especially involving repeated, low value, and novel transactions, such as food delivery [8]. Platforms focus on attracting customers, whose demand would in turn attract suppliers, leading to network-effects based winner-take-all dynamics [27, 28]. Thus, platform participation has been an area of emphasis in the literature [29-31]. In particular, service participation on food delivery digital platforms can bring a lot of benefits to restaurants (in the form of increased orders and revenue); at the same time, if restaurants are unable to cope with the increased demands, their online reputation (i.e., their online ratings) is also likely to suffer irreparable losses. Service participation is thus a strategic choice for suppliers.

Two relevant thematic issues emerge from our review of the extant literature. First, prior research has focused primarily upon inter-platform competition and strategic behavior of platform providers, with few notable exceptions [8, 19]. Our examination adds to a nascent stream of literature. Second, while there are digital platforms have been defined variously, not many previous studies have conceptualized digital platforms as service systems.

\subsection{Service Assortment}

The performance effects of assortment size have been an enduring research topic in marketing, information systems [1,12], and operation management. Three key thematic issues emerge from our review of this literature. First, assortment has mixed effects on customers as though customers seek larger variety, they get overwhelmed as assortment increases. The former is because assortment increases the probability of finding ideal products [32], thereby signals higher variety and therefore satisfies variety-seeking needs [33] [34]. The latter occurs because large assortments are cognitively demanding [33] and result in confusion and frustration [35]. Eventually, large assortments dissatisfy customers [36], leading to detrimental impacts on sales. However, assortment also has mixed effects on sales and performance. While reduction in assortment has a negative effect on purchase quantity [37], it also enhances sales of certain items [38]. Our research attempts to address this issue by examining the influence of assortment in consort with other information attributes that may concurrently influence performance.

Second, most previous studies on assortment have focused on offline stores, barring a few exceptions that have examined online merchants (e.g., [10]). However, these examinations have exclusively considered one or the other, unlike our study wherein we simultaneously study service providers who operate exclusively offline and service providers who operate both offline and online through the digital platform. Given our unique setting, we are able to examine the implications of the strategic choices (e.g., service assortment in the form of cuisine variety and service participation on the food delivery service offered by the platform) made by restaurants for their performance on the food delivery digital platform.

Third, prior research has largely focused on conceptualizing assortment as the number of options offered in a single product category. However, assortment can also be conceptualized as the number of product categories [9] and is a more apt measure as the number of categories offered is a prerequisite for determining the number of options offered as a whole. Furthermore, given that customers choose a certain product category before they choose a specific item within that category, this conceptualization is more aligned to customer behavior [39]. We add to this emergent stream of literature.

\subsection{Ratings}

It is also pertinent for us to review related literature on ratings because ratings are the dependent variable of our analysis $[1,12]$. User-generated ratings have a strong influence on the behavior of other users because customers use ratings to simplify (reduce) the amount of effort that they expend on making decisions regarding selection and purchase. Formally, ratings reduce information asymmetry by soliciting and displaying information about transaction quality to market participants. Hence ratings can improve efficiency and overcome market failure [40]. Ratings matter, but not universally [41, 42]. While some find no effect of ratings on sales, others find negative and significant 
effects. Also, the nature of the product or service being rated and the nature of the rating system (one-sided versus two-sided ratings) matter as they influence the distribution and consequences of ratings. In sum, ratings encapsulate and reflect performance.

\section{Methods}

We integrate induction and abduction for theory development. Induction is a machine learning methodology for discovering patterns from big data [19]. After discovering patterns in data, we make sense of the induced patterns by abductively developing the most viable, generalizable explanations. In summary, abduction is an approach to theory building that completes the knowledge production process by making sense of the data-driven patterns discovered by induction [13].

\subsection{Research context}

India is an apt setting for examining our research question. A largely agrarian society, India has been one of the fastest growing major economies in the world [21]. This has led to the rise of a large group of consumers who aspire to western products and services [43], resulting in a transitional economy [22, 44, 45]. Specifically, our research context is a large, comprehensive restaurant discovery and food delivery digital platform based in India. This platform has a panIndia presence and has been in operation for more than 3 years. This platform comprises of two distinct elements: a website to review and rate restaurants, and a separate online marketplace for ordering and delivering food. All registered restaurants in India are listed on the website, irrespective of whether they participate in food delivery or not. Thus, all restaurants receive ratings (subject to a few conditions elaborated upon at the end of this section). This effectively addresses concerns stemming from sample selection bias as we are able to observe ratees, irrespective of whether they participate in the marketplace or not. In our setting, multi-homing costs are low and a restaurant can choose to affiliate with any number of digital platforms. Research suggests that winner-take-all outcomes are unlikely in such domains [46].

We started with a population sample of 95,735 restaurants, serving a total of 135 different cuisines, located in 37 cities of India as our dataset. Restaurants across India are part of the sample if they are listed on the digital platform. Any consumer can list a restaurant on the website; listed restaurants can garner reviews and ratings from other consumers. The restaurant owner must claim the listing if she wishes to provide verified details such as an official menu, contact information and opening hours. Restaurants with missing ratings were excluded from the analysis. We also chose restaurants only from the top seven metros in India (Mumbai, Delhi, Chennai, Kolkata, Pune, Hyderabad and Bangalore) as cities outside the top seven metros are not likely to appreciate international cuisines.

Since induction yields easy-to-interpret decision rules [47] organized in a tree, this user-friendly methodology is often preferred by top management executives. Induction opens up the black box of decision making and represents emergent interrelationships between decision attributes and outcomes (information attributes are inputs to induction; outputs of induction, namely the attributes included in the tree, are referred to as decision attributes) [8].

Induction makes relatively few distributional assumptions about the data thereby making this methodology generalizable. Data partitioning creates non-overlapping training and validation partitions necessary for ascertaining the generalizability of knowledge. Knowledge is discovered from the training partition and validated using unseen data from the validation partition. In this study, we use 10-fold validation via data partitioning for avoiding the overfitting trap. In summary, we assess generalizability of the knowledge discovered on training data by testing its prediction accuracy on unseen data from the validation data partition. Following up induction with abduction is vital for theory development as it enables us to develop generalizable explanations for making sense of the data-driven patterns induced from big data.

\subsection{Data: Modeling customer journeys}

We investigate customer journeys as they rate restaurants on the digital platform $[1,12]$. Customers can either choose (home delivery) convenience by having the platform deliver food to their homes or choose the (restaurant dining) experience. We investigate the customer journeys in both these distinct scenarios. The outcome variable, rating, is coded as high / (low) if the restaurant rating is above / (below) the mean. Next, we describe information attributes included in our theory.

The first key attribute we included was the price range. The cost of a meal for two persons reflects the strategic positioning of the restaurant (cost leadership $[48,49]$. Specifically, a restaurant that offers a meal for two persons for $1000 \mathrm{INR}$ and above was assigned a value of high price range (approximately 14 US Dollars). The cost for a restaurant that offers a meal less than or equal to 300 INR (approximately 4 US Dollars) assigned a value of low. Restaurants where a cost for a meal was between 300 and 1000 INR was considered in the medium price range. 
Cuisine Variety was assigned a value of low if the restaurant offered a single cuisine, medium if two or three cuisines were offered. A value of high was assigned to this variable if the restaurant offered more than three cuisines.

If the restaurant is a vegetarian only restaurant or not is captured by using a dummy called Vegetarian. A value of Yes was assigned to this attribute if the restaurant was a vegetarian only restaurant, otherwise a value of No assigned to this attribute.

Similarly, if the restaurant provides only Indian food (vs. world cuisines) is captured using a dummy called Only Indian. A value of Yes was assigned to this attribute if the restaurant serves only Indian food, otherwise a value of No assigned to this attribute.

If the restaurant serves alcohol is captured using a dummy called Alcohol. A value of Yes was assigned to this attribute if the restaurant serves alcohol, otherwise a value of No assigned to this attribute.

A key institutional attribute that we captured corresponds to whether a restaurant is part of a group of restaurants; a restaurant chain reflected by the same or similar names. These restaurants may be part of a chain or might share a common name that reflects a wellestablished "institutional" identity (e.g., [50]). Thus, we capture this attribute by assigning Institutional Chain a value of high if nine or more other restaurants had the same name as the focal restaurant. A value of medium is assigned at least one other restaurant, and less than nine other restaurants, shared their names with the focal restaurant. If the restaurant's name was unique, low value is assigned.

From the point of view of the customer, another type of variety is captured in the notion of market or competitive density.

Table 1. Understanding customer journeys

\begin{tabular}{|c|l|l|}
\hline NO. & ATTRIBUTES & \multicolumn{1}{c|}{ VALUES } \\
\hline \multicolumn{2}{|c|}{ Information attributes for explaining ratings } \\
\hline 1 & Price Range & $\begin{array}{l}\text { Low: cost of meal for two persons } \\
\text { less than 300 Indian Rupees } \\
\text { [INR] (approx. 4 USD); Medium: } \\
\text { cost of meal for two persons } \\
\text { between 300 and 1000 INR; High: } \\
\text { cost of meal for two persons } \\
\text { greater than 1000 INR (approx. 14 } \\
\text { USD) }\end{array}$ \\
\hline 2 & Cuisine Variety & $\begin{array}{l}\text { Low: restaurant serves a single } \\
\text { cuisine; Medium: restaurant } \\
\text { serves two or three cuisines; } \\
\text { High: restaurant serves more than } \\
\text { three cuisines }\end{array}$ \\
\hline 3 & Vegetarian & $\begin{array}{l}\text { No: restaurant serves non- } \\
\text { vegetarian food / Yes: restaurant } \\
\text { serves only vegetarian food }\end{array}$ \\
\hline
\end{tabular}

\begin{tabular}{|c|l|l|}
\hline 4 & Only Indian & $\begin{array}{l}\text { No: restaurant serves non-Indian } \\
\text { cuisines / Yes: restaurant serves } \\
\text { only Indian cuisines }\end{array}$ \\
\hline 5 & Serves Alcohol & $\begin{array}{l}\text { No: restaurant does not serve } \\
\text { alcohol / Yes: restaurant serves } \\
\text { alcohol }\end{array}$ \\
\hline 6 & $\begin{array}{l}\text { Institutional } \\
\text { Chain } \\
\text { institutional chain; Medium: less } \\
\text { than nine other restaurants with a } \\
\text { similar name; High: more than } \\
\text { nine restaurants with a similar } \\
\text { name }\end{array}$ \\
\hline 7 & $\begin{array}{l}\text { Restaurant } \\
\text { Variety }\end{array}$ & $\begin{array}{l}\text { within the 1-kilometre distance of } \\
\text { this restaurant; Medium: between } \\
\text { 10 and 99 within the 1-kilometre } \\
\text { distance of this restaurant; High: } \\
\text { More than 100 restaurants within } \\
\text { the 1-kilometre distance of this } \\
\text { restaurant }\end{array}$ \\
\hline $\begin{array}{l}\text { Decision Outcome: Customer Rating Journeys } \\
\text { Scenario 1: (Home Delivery) Convenience: Restaurants } \\
\text { participate in food delivery } \\
\text { Scenario 2: (Restaurant Dining) Experience: Restaurants } \\
\text { don't participate in food delivery }\end{array}$ \\
\hline Ratings & $\begin{array}{l}\text { Low: restaurant rating less than } \\
\text { the mean; High: restaurant rating } \\
\text { greater than the mean }\end{array}$ \\
\hline
\end{tabular}

Accordingly, restaurant variety (calculated for each focal restaurant) is captured by density of restaurants relative to a focal restaurant. Restaurant variety which represents spatial concentration of competition was calculated for each focal restaurant as the number of restaurants that lie within 1-kilometre distance of that focal restaurant. Restaurant variety variable was assigned three values. We assigned a value of low if number of restaurants was $<9$, medium if number of restaurants was between 10 and 99 (both inclusive) and high if number of restaurants that lie within 1-kilometre distance of that focal restaurant was $>=$ to 100 .

The two scenarios of interest; (home delivery) convenience vs. (restaurant dining) experience were captured based on the restaurant's participation on the food delivery component of the digital platform. Restaurants that did not participate on the delivery platform were grouped together to study the (restaurant dining) experience scenario. Restaurants that participated on delivery platform were grouped together to study the (home delivery) convenience scenario.

Finally, the focal variable of our analysis, a restaurant's online rating was captured. A restaurant's online rating represents its reputation or social capital in the digital world. A restaurant's offline reputation migrates to the digital platform as more and more customers review and rate the restaurant. Overall, since information contained in the reviews is distilled to one final online rating, we only included the overall online 
rating in our analysis. This website recorded a restaurant's rating on a 5-point scale. We transformed ratings from their numeric value to two categories of high and low as follows. A restaurant is coded to have high rating when its rating is greater than or equal to the mean rating. A restaurant is coded to have low rating when its rating is less than the mean rating.

35,815 restaurants did not have ratings and such restaurants were excluded from our analysis. We observe that large number of the missing ratings in our population belong to restaurants that have gone out of business. Newly opened restaurants do not have a rating for the first 3-months due to website regulations. Restaurants that have not accumulated a significant number of reviews also do not have a rating. We conducted detailed ex post analysis on this sub-sample. In small cities, the platform is arguably not used for reviews. A majority of restaurants with missing ratings had low prices; these restaurants plausibly do not have enough reviews because their customers, arguably, may not be technology savvy. Overall, we can infer that half of the restaurants without ratings did not have enough reviews, whereas the other half were either closed or newly opened. In summary, restaurants without ratings were excluded from our main analyses.

\subsection{Induction and Validation with Prediction}

Two steps define knowledge discovery via induction $[1,12]$. First, the C4.5 algorithm is used to induce a decision tree on training data [15]. Second, tree grown in step 1 is pruned by validating it with unseen data from the validation partition. By employing high levels of pruning, we discover the tacit structure of data and demonstrate robustness of discovered knowledge. The Weka software application, an open-source platform is used for data partitioning, and for growing and pruning trees [51]. The $\mathrm{C} 4.5$ algorithm relies on the concept of purity and utilizes informative attributes to recursively partition the training data to reduce impurity in terminal nodes. Entropy is chosen as the impurity measure, as entropy is easy to interpret for a two-class decision problem $[15,16]$.

Tree induction iteratively groups together observations (i.e., restaurants) such that they are similar not only in certain information attributes (information attributes from Table 1) but also similar in terms of their ratings. There are two inputs to tree induction: (1) restaurants described by all information attributes (as described in Table 1), and (2) restaurant ratings. We investigate the customer journeys for ratings in two different scenarios of (home delivery) convenience vs (restaurant dining) experience. The objective of tree induction is to discover tacit combinations of information attributes associated with similar final outcomes (i.e., similar ratings) [15]. Trees only retain the most pertinent attributes for explaining decisions and organize decision attributes in a context-dependent manner; certain questions are only raised depending on answers obtained to other questions [16].

Trees discovered by induction are not reflective of the exact rules or "scripts", but rather represent credible approximations of customer journeys. Instead of the correlations between attributes, induction relies on the amount of information a particular attribute conveys about the decision outcome.

To ensure that decision rationale is comprehensively discovered, a process of drawing mutually exclusive, training and testing subsamples is repeated multiple times. In each iteration, we draw random, mutually exclusive subsamples of restaurants from the original data; one set, known as the training set, from which the tacit decision rationale is discovered by the C4.5 induction algorithm [15], and another disjoint set of initiatives, known as the testing set, which is used to test the predictive accuracy of this discovered rationale. We used 10-fold validation where the full sample is divided into 10 partitions of which 9 partitions are used for building the tree and the last partition is used for validation. Prediction accuracy of the tree discovered from training set is assessed by predicting decisions from unseen data from the validation set.

Multiple approximations of the tacit rationale are derived by conducting computational experiments whereby the 10 -fold validation process is repeated at varying levels of pruning. Using prediction accuracy of the decision tree as the sole criterion when choosing the best representative tree (among alternative models) can be misleading and would be akin to falling into the overfitting trap. We rely on three heuristics [47], namely (i) high prediction accuracy, (ii) high parsimony and (iii) high reliability to select the best representative trees across the two scenarios of (home delivery) convenience vs (restaurant dining) experience. Thus, we are fairly certain that trees presented here are the "best", most credible approximations of the customer journeys for rating restaurants on the platform.

All seven information attributes characterizing restaurants (see Table 1) across the two scenarios of (home delivery) convenience vs (restaurant dining) experience in conjunction with the final restaurant rating are inputs to induction. All information attributes deemed informative for explaining ratings are included in the trees as decision attributes and the induction algorithm excludes all the non-informative attributes from the tree. The most informative decision attribute is the top-most attribute in the tree. Importance of attributes decreases as we move away from the top of the tree to its leaves. Trees organize attributes in a 
context-dependent manner; certain questions are only raised depending on answers obtained to questions answered previously [16].

\subsection{Key findings from induction}

The key findings from induction are presented next. Assortment in the form of restaurant variety matters and enables superior performance in the form of higher ratings. When consumers enjoy home delivery convenience (see Figure 1), restaurants that serve alcohol perform poorly and consumers rate them poorly. When consumers want to enjoy home delivery convenience, assortment in terms of high restaurant variety matters as that provides consumers the ability to pick and choose their food from a variety of restaurants.

\begin{tabular}{|c|}
\hline $\begin{array}{l}\text { How customers rate restaurants that } \\
\text { deliver food using the online platform }\end{array}$ \\
\hline Restaurant Variety = Low: Low Ratings \\
\hline Restaurant Variety $=$ Medium \\
\hline Only Indian $=$ No \\
\hline Serves Alcohol $=$ No \\
\hline Price Range $=$ Low \\
\hline | Vegetarian $=$ No: Low Ratings \\
\hline | Vegetarian $=$ Yes: High Ratings \\
\hline Price Range $=$ Medium: High Ratings \\
\hline Serves Alcohol = Yes: Low Ratings \\
\hline Only Indian $=$ Yes: Low Ratings \\
\hline Restaurant Variety $=$ High: High Ratings \\
\hline
\end{tabular}

Figure 1: Customer Convenience

When consumers enjoy the restaurant dining experience (see Figure 2), assortment matters again as higher (in restaurant) cuisine variety is associated with superior performance (in the form of higher restaurant ratings).

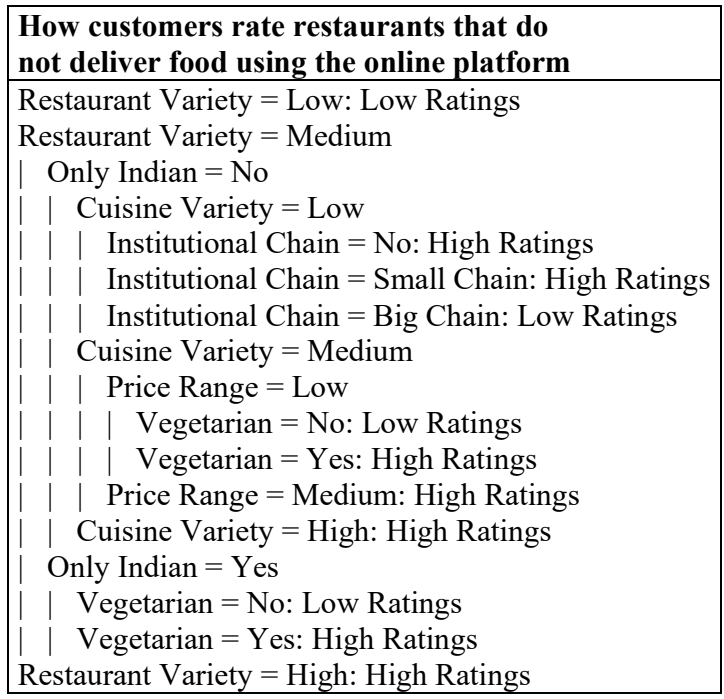

Figure 2: Customer Experience

\subsection{Abducting away: Generalizable Explanations for Customer Journeys}

Induction yields a collection of rules [8, 19, 52, 53]. For the two distinct scenarios of home delivery convenience and restaurant dining experience, we selected one best representative tree. Abduction is the systematic process of arriving at the most plausible explanation of the discovered rules [8, 19]. We followed the following steps to abduct away from the two best representative trees presented in Figure 1 and Figure 2 respectively.

We conducted a systematic analysis of the two trees to identify key similarities and key differences. These similarities and differences are summarized in Table A1 in the appendix. We find that assortment, i.e. restaurant variety matters across the board and cuisine variety matters when consumers choose the restaurant dining experience.

A deeper understanding of the rules can only emerge as we synthesize a rich understanding of the context by integrating the viewpoints of the customers (who rate the restaurants) and the restaurants (that are being rated by the customers). From the point of view of restaurants, the restaurants have access to two main levers which they can adjust to enhance their market orientation. Restaurants can either (i) adjust their cuisine offerings or they can (ii) participate or not participate on the platform. We find that restaurant variety matters in both the contexts of home delivery convenience and restaurant dining experience. Cuisine variety in particular, matters more in the context of restaurant dining experience. Abducting away, we find variety is indeed the spice of life (and ratings) and enables superior performance in the form of higher ratings.

\section{Discussion and concluding comments}

This manuscript makes three critical contributions to theory. First, trees represent theories in a holistic and easy to interpret manner [8]. Hence our trees and the findings from them represent contributions to extant literature. Second, we enhance the literature on digital platforms by highlighting the role of variety assortment and market density, on the performance of service providers who participate on platforms [8, 19]. Third, we contribute to the assortment literature by examining a hybrid case of service providers who are both offline and online. Finally, our results also have implications for literature on service systems [7].

From a practice perspective, our findings illuminate a prescription for service providers who are considering participating on digital platforms. Given the accelerating trend towards convenience as an organizing principle, most service providers will be tempted to 
participate on digital platforms. However, market density is exogenous, and hence if the provider is present in a location with low market density, perhaps a more prudent choice would be to not participate on the digital platform and instead focus upon experience seeking customers by increasing category assortment.

Our results must be considered while keeping in mind limitations of our study. First, decision trees are approximations, of the decision-making process tacitly followed by customers. Second, generalizability of our results to other types of platforms may be limited. Even though our theory is generalizable to all platforms, it is plausible that the results not apply to all types of current and future platforms. Finally, cross-sectional nature of our data precludes us from drawing causal conclusions through our analysis.

\section{References}

[1] P. Karhade and J. Dong, "Innovation Outcomes of Digitally Enabled Collaborative Problemistic Search Capability," MIS Quarterly, Forthcoming, 2020.

[2] P. Karhade and J. Dong, "Information Technology Investment and Commercialized Innovation Performance: Dynamic Adjustment Costs and Curvilinear Impacts," MIS Quarterly, Forthcoming, 2020.

[3] J. Q. Dong, J. He, and P. Karhade, "The Penrose effect in resource investment for innovation: Evidence from information technology and human capital," ECIS, 2013.

[4] J. Q. Dong, P. Karhade, A. Rai, and S. X. Xu, "Information Technology in Innovation Activity of the Firm: Theory and Synthesis," ECIS, 2013.

[5] J. Q. Dong, P. Karhade, A. Rai, and X. S. Xu, "How Firms Make Information Technology Investment Decisions: Toward a Behavioral Agency Theory," Journal of Management Information Systems, Forthcoming, 2020.

[6] T. J. V. Saldanha, A. Sahaym, S. Mithas, M. G. Andrade-Rojas, A. Kathuria, and H.-H. Lee, "Turning Liabilities of Global Operations into Assets: IT-Enabled Social Integration Capacity and Exploratory Innovation," Information Systems Research, vol. 31, no. 2, pp. 361-382, 2020, doi: 10.1287/isre.2019.0890.

[7] T. Saldanha, A. Kathuria, J. Khuntia, and B. Konsynski, "Ghosts in the Machine: How Marketing and Human Capital Investments Enhance Customer Growth when Innovative Services Leverage Self-Service Technologies," Information Systems Research, forthcoming.

[8] A. Kathuria, P. P. Karhade, and B. R. Konsynski, "In the Realm of Hungry Ghosts: Multi-Level Theory for Supplier Participation on Digital Platforms," Journal of Management Information Systems, vol. 37, no. 2, pp. 396430, 2020.

[9] Y. He, X. Guo, and G. Chen, "Assortment Size and Performance of Online Sellers: An Inverted U-Shaped Relationship," Journal of the Association for Information Systems, vol. 20, no. 10, p. 2, 2019.

[10] J. Ma, "Does greater online assortment pay? An empirical study using matched online and catalog shoppers," Journal of Retailing, vol. 92, no. 3, pp. 373-382, 2016.
[11] N. Hashai, "Within-industry diversification and firm performance-an S-shaped hypothesis," Strategic Management Journal, vol. 36, no. 9, pp. 1378-1400, 2015.

[12] M. L. Markus, A. Majchrzak, and L. Gasser, "A design theory for systems that support emergent knowledge processes," MIS quarterly, pp. 179-212, 2002.

[13] V. Grover, A. Lindberg, I. Benbasat, and K. Lyytinen, "The Perils and Promises of Big Data Research in Information Systems," Journal of the Association for Information Systems, vol. 21, no. 2, p. 9, 2020.

[14] J. G. March, Primer on decision making: How decisions happen. Simon and Schuster, 1994.

[15] J. R. Quinlan, "Induction of decision trees," Machine learning, vol. 1, no. 1, pp. 81-106, 1986.

[16] J. R. Quinlan, "Decision trees and decisionmaking," IEEE Transactions on Systems, Man, and Cybernetics, vol. 20, no. 2, pp. 339-346, 1990.

[17] C. S. Peirce, Collected papers of charles sanders peirce. Harvard University Press, 1974.

[18] J. Reichertz, "Abduction: The logic of discovery of grounded theory," The SAGE handbook of grounded theory, pp. 214-228, 2007.

[19] P. P. Karhade and A. Kathuria, "Missing Impact of Ratings on Platform Participation in India: A Call for Research in G. R. E. A. T. Domains," Communications of the Association for Information Systems, vol. 47, 2020.

[20] A. Kathuria, A. Mann, J. Khuntia, T. Saldanha, and R. J. Kauffman, "A Strategic Value Appropriation Path for Cloud Computing," Journal of Management Information Systems, vol. 35, no. 3, pp. 740-775, 2018.

[21] R. Kathuria, N. N. Kathuria, and A. Kathuria, "Mutually supportive or trade-offs: An analysis of competitive priorities in the emerging economy of India," The Journal of High Technology Management Research, vol. 29, no. 2, pp. 227-236, 2018.

[22] J. Khuntia, A. Kathuria, T. J. V. Saldanha, and B. R. Konsynski, "Benefits of IT-Enabled Flexibilities for Foreign versus Local Firms in Emerging Economies," Journal of Management Information Systems, vol. 36, no. 3, pp. 855892, 2019/07/03 2019, doi: 10.1080/07421222.2019.1628906.

[23] P. A. Pavlou and A. Dimoka, "The Nature and Role of Feedback Text Comments in Online Marketplaces: Implications for Trust Building, Price Premiums, and Seller Differentiation," Information Systems Research, vol. 17, no. 4, pp. 392-414, 2006, doi: 10.1287/isre.1060.0106.

[24] S. L. Vargo and R. F. Lusch, "Evolving to a new dominant logic for marketing," Journal of marketing, vol. 68, no. 1, pp. 1-17, 2004.

[25] J. Spohrer, P. P. Maglio, J. Bailey, and D. Gruhl, "Steps toward a science of service systems," Computer, vol. 40, no. 1, pp. 71-77, 2007.

[26] S. Vitzthum, A. Kathuria, and B. Konsynski, "Toys become Tools: From Virtual Worlds to Real Commerce," Communications of the Association for Information Systems, vol. 29, no. 1, p. 21, 2011.

[27] Y. Bakos and E. Katsamakas, "Design and ownership of two-sided networks: Implications for Internet platforms," Journal of Management Information Systems, vol. 25, no. 2, pp. 171-202, 2008. 
[28] C. Cennamo and J. Santalo, "Platform competition: Strategic trade-offs in platform markets," Strategic Management Journal, vol. 34, no. 11, pp. 1331-1350, 2013.

[29] K. J. Boudreau and L. B. Jeppesen, "Unpaid crowd complementors: The platform network effect mirage," Strategic Management Journal, vol. 36, no. 12, pp. 17611777, 2015, doi: doi:10.1002/smj.2324.

[30] A. Ghose and P. G. Ipeirotis, "Estimating the helpfulness and economic impact of product reviews: Mining text and reviewer characteristics," IEEE Transactions on Knowledge and Data Engineering, vol. 23, no. 10, pp. 1498-1512, 2011.

[31] D. P. McIntyre and A. Srinivasan, "Networks, platforms, and strategy: Emerging views and next steps," Strategic Management Journal, vol. 38, no. 1, pp. 141-160, 2017.

[32] D. Kuksov and J. M. Villas-Boas, "When more alternatives lead to less choice," Marketing Science, vol. 29, no. 3 , pp. 507-524, 2010.

[33] A. Chernev and R. Hamilton, "Assortment size and option attractiveness in consumer choice among retailers," Journal of Marketing Research, vol. 46, no. 3, pp. 410-420, 2009.

[34] L. McAlister and E. Pessemier, "Variety seeking behavior: An interdisciplinary review," Journal of Consumer research, vol. 9, no. 3, pp. 311-322, 1982.

[35] B. E. Kahn, E. Weingarten, and C. Townsend, "Assortment variety: Too much of a good thing?," in Review of Marketing Research: Emerald Group Publishing Limited, 2013.

[36] K. Diehl and C. Poynor, "Great expectations?! Assortment size, expectations, and satisfaction," Journal of Marketing Research, vol. 47, no. 2, pp. 312-322, 2010.

[37] S. Borle, P. Boatwright, J. B. Kadane, J. C. Nunes, and S. Galit, "The effect of product assortment changes on customer retention," Marketing science, vol. 24, no. 4, pp. 616-622, 2005.

[38] P. Boatwright and J. C. Nunes, "Reducing assortment: An attribute-based approach," Journal of marketing, vol. 65, no. 3, pp. 50-63, 2001.

[39] A. G. Kök and Y. Xu, "Optimal and competitive assortments with endogenous pricing under hierarchical consumer choice models," Management Science, vol. 57, no. 9, pp. 1546-1563, 2011.

[40] A. Pallais, "Inefficient Hiring in Entry-Level Labor Markets," American Economic Review, vol. 104, no. 11, pp. 3565-99, 2014, doi: doi: 10.1257/aer.104.11.3565.

[41] W. Duan, B. Gu, and A. B. Whinston, "Do online reviews matter? - An empirical investigation of panel data," Decision Support Systems, vol. 45, no. 4, pp. 10071016, 2008/11/01/ 2008, doi: https://doi.org/10.1016/j.dss.2008.04.001.

[42] Y.-J. Lee, K. Hosanagar, and Y. Tan, "Do I Follow My Friends or the Crowd? Information Cascades in Online Movie Ratings," Management Science, vol. 61, no. 9, pp. 2241-2258, 2015, doi: 10.1287/mnsc.2014.2082.

[43] N. Celly, A. Kathuria, and V. Subramanian, "Overview of Indian multinationals," Emerging Indian Multinationals: Strategic Players in a Multipolar World, 2016.
[44] J. Khuntia, A. Kathuria, M. Andrade Rojas, T. Saldanha, and N. Celly, "How Foreign and Domestic Firms Differ in Leveraging IT-enabled Supply Chain Information Integration in BOP Markets: The Role of Supplier and Client Business Collaboration," Journal of the Association for Information Systems, forthcoming.

[45] J. Khuntia, A. Kathuria, T. Saldanha, and B. Konsynski, "Benefits of IT-enabled Flexibilities for Foreign versus Local Firms in Emerging Economies," Journal of Management Information Systems, vol. 36, no. 3, pp. 1-38, 2019.

[46] A. Hagiu, "Two-Sided Platforms: Product Variety and Pricing Structures," Journal of Economics \& Management Strategy, vol. 18, no. 4, pp. 1011-1043, 2009, doi: doi:10.1111/j.1530-9134.2009.00236.x.

[47] P. Karhade, M. J. Shaw, and R. Subramanyam, "Patterns in Information Systems Portfolio Prioritization: Evidence from Decision Tree Induction," Mis Quarterly, vol. 39, no. 2, 2015.

[48] M. E. Porter, "Competitive strategy: Techniques for analyzing industries and competition," New York, vol. 300, p. $28,1980$.

[49] M. E. Porter and V. E. Millar, "How information gives you competitive advantage," ed: Harvard Business Review Juli/August, 1985.

[50] M. T. Hannan and J. Freeman, "The population ecology of organizations," American journal of sociology, vol. 82, no. 5, pp. 929-964, 1977.

[51] M. Hall, E. Frank, G. Holmes, B. Pfahringer, P. Reutemann, and I. H. Witten, "The WEKA data mining software: an update," $A C M$ SIGKDD explorations newsletter, vol. 11, no. 1, pp. 10-18, 2009.

[52] P. Karhade and M. Shaw, "Rejection and Selection Decisions in the IT Portfolio Composition Process: An Enterprise Risk Management Based Perspective," Americas Confrence on Information Systems, p. 221, 2007.

[53] P. P. Karhade, M. J. Shaw, and R. Subramanyam, "Evolution of Decision Rules used for IT Portfolio Management: An Inductive Approach," in Americas Conference on Information Systems, 2009: Springer, pp. 307-320. 


\section{Appendix}

\section{Table A1. Drawing abductive insights}

\begin{tabular}{|c|c|}
\hline (Restaurant Dining) Experience & (Home Delivery) Convenience \\
\hline Key Similarities & \\
\hline \multicolumn{2}{|l|}{$\begin{array}{l}\text { Attributes that detine the customer journeys } \\
\begin{array}{ll}\text { 1. } & \text { Restaurant variety } \\
\text { 2. } & \text { Only Indian cuisines } \\
\text { 3. } & \text { Vegetarian Only } \\
\text { 4. } & \text { Price range }\end{array}\end{array}$} \\
\hline \multicolumn{2}{|l|}{ Key Differences } \\
\hline Variety of cuisines in the restaurant & If the restaurant serves alcohol \\
\hline \multicolumn{2}{|c|}{$\begin{array}{l}\text { Restaurant variety matters! } \\
\text { Consistently" we found that when customers have more choices, in terms of more restaurants, they award higher ratings to } \\
\text { restaurants located in a high density of restaurants. The density of variety matters! In that sense, variety is the spice of ratings! } \\
\text { Additionally: When customers choose the restaurant dining experience; variety and more choices of cuisines matter when } \\
\text { customers are in the restaurant; especially, when restaurants choose not to deliver food using the platform. } \\
\text { Variety is the spice of life and ratings! }\end{array}$} \\
\hline $\begin{array}{l}\text { Cuisine variety is good for ratings when customers choose } \\
\text { the restaurant dining experience! }\end{array}$ & $\begin{array}{l}\text { When customers choose home delivery convenience, } \\
\text { restaurants that serve alcohol are not preferred and in fact, } \\
\text { their online ratings can suffer! }\end{array}$ \\
\hline $\begin{array}{l}\text { If you are a part of a big chain, that is likely to hurt your } \\
\text { online ratings! }\end{array}$ & $\begin{array}{l}\text { If you only serve Indian food; that is likely to hurt your } \\
\text { online ratings. Everyone always eats desi food at home; can } \\
\text { be more critical? }\end{array}$ \\
\hline
\end{tabular}

